\title{
Proposing a Model for Analyzing Effects of Media Literacy Components on Improvement of Social Skills and Moderating Impact of Cultural Capital Based on Structural Equations Model Approach
}

\author{
Ali Shahsavari \\ Master Student, Department of Humanities Sciences, Isfahan (Khorasgan) Branch, Islamic Azad University, Isfahan, Iran \\ Email: a.shahsavary@gmail.com

\section{Reza Ebrahimzadeh Dastjerdi}

Faculty Member, Department of Humanities Sciences, Isfahan (Khorasgan) Branch, Islamic Azad University, Isfahan, Iran

\author{
Doi:10.5901/mjss.2016.v7n3s3p376
}

\begin{abstract}
The objective of the current paper is to analyze the relationship between media literacy and cultural capital and social skills among residents of Isfahan City. The research methodology is based on descriptive correlation technique. The statistical population of the present research comrpises all citizens of Isfahan City (1,796,967 persons). Using Cochran's sampling formula, 384 individuals were selected as sample size via stratified sampling method. Research tool consists of three questionnaires two of which were standard scales and the third one was designed by the researcher. The questionaires include: Karaman\&Karatash's Standard Questionnaire of Media Literacy (2009), Oldmeadow et al's Standard Questionnaire of Social Skills, and the researcher-made questionnaire of cultural capital based on Bourdieu's theory whose validity was confirmed via both content and factor analyses. Based on Cronbach's alpha coefficient, reliability coeficients of Media Literacy Questionnaire, Scoial Skill Questionnaire, and Cultural Capital Questionnaire were respectively estimated to be $0.876,0.896$, and 0.913. Data were analyzed at two levels: descriptive (mean and frequency table) and inferential (structural equations modeling). The research results indicated that possession of media literacy affects social skill with an effect size of 0.37 and cultural capital moderates the relationship with an effect size of 0.17 . On the other hand, the research results showed that experience and analysis and reaction affect social skills with an effect size of 0.68 but cultural capital moderates the relationship with an effect size of 0.2. Results of the third hypothesis demonestrated that judgement and observed hidden message influences social skills with an effect size of 0.53 and cultural capital moderates this effect with an effect size of 0.2 . Finally, the relationships among variables were depicted, and based on research findings, recommendations were made to improve media literacy, social skills, and cultural capital of Isfani citizens.
\end{abstract}

Keywords: Social Skills, Cultural Capital, Analysis of Hidden Messages, Media literacy, Judgement and Observed Hidden Message

\section{Introduction}

In the modern world, power indexes have undergone a drastic shift to such an extent that culture is regarded as the most significant infrastructural element in formation of characteristic dimensions of person, group, and society. Media can be mentioned as one of the most powerful means of culture. Following birth of printing industry, these means have exceedingly taken up a place in all affairs of human life, and with establishment of mass media, they have advanced to present themselves as the strongest man-made tool. Now, rigorous and efficient solutions shall be explored in order to make a connection with this great power. And there is no solution but media literacy. Media literacy is a regular framework in establishing a useful and effective communication with the media that have conquered almost the whole human life. Social relationships of humans have expanded extraordinarily in the present era when the media have surpassed the concept of time and space dimensions. The expansion is so remarkable that acquisition of social skills is required to properly manage the circumstances. Media literacy coupled with appropriate and meticulous management of utilization and application of media as the most important means of communication among people can influence establishment of social skills.

On the other hand, cultural capital as the cultural assets accumulated by the course of time has an effect on provision of this context and affects the relationship between media literacy and social skills. It is therefore attempted in the present research to propose a model for analyzing effect of media literacy components on improvement of social 
skills as well as moderating effect of cultural capital based on the approach of structural equations model.

\subsection{Problem Statement}

Advent of media literacy concept dates back to 1965. McLuhan coined the term for the first time in his book "Understanding Media: The Extensions of Man" .McLuhan believed when the age of global village arrives, the humans shall achieve a new sort of literacy called "media literacy" (Banai, 2006). Media literacy emphasizes on the fact that understanding media messages and mechanism of behavior with the media, unlike the commonplace presumption, requires special knowledge and skills. And in more precise words, it constitutes skills of reasoning thought and critique such that the addressee can have an independent judgment about the media content (Arjmand, 2005:5). Different theorists have proposed varying attitudes about constituting dimensions of media literacy. Based on the attitudes of Karaman \& Karatash (2009). Media literacy dimensions include the followings:

Media Knowledge (Critical Thinking) includes a set of skills that a person acquires and can easily critique the messages rendered by mass communication media and react to accuracy or inaccuracy of the messages existing in the media, and also, recognize the political, economic, cultural, and social orientations of mass media (Karaman \& Karatash 2009; p: 799).

Judgment and Observed Hidden Messages: refers to the set of capabilities and knowledge based on which the addressee of media messages can understand the impact of financial sponsors and supporters of media programs on their messages and is able to easily comprehend and realize the latent and implicit messages transmitted via mass media.

Analysis and Reaction (to media messages): refers to the set of skills and abilities by means of which the media addressee is able to recognize the biased messages of mass media and to react appropriately to harmful messages exposed by mass communication media (Karaman \& Karatash 2009; page 801).

The respective dimensions might influence communicational features of individuals and their social skills. Many definitions have been proposed for social skills structure. Some of the definitions include: social skills are the skills that allow the person to start and resume positive interactions with others and comprises skills such as communicationestablishing, problem-solving, decision-making, self-management, and relations with peers (Seven \& Yoldas, 2007; Chritophersen \& Vanscoyoc 2008 quoted by Aydogan et al., 2009). Matson et al. (2003) defined social skills as observable and measurable behaviours which improve independence, acceptance, and desirable life quality. The respective skills are important for compatibility and normal function, and, disorder in social skill is closely associated with mental disorders and behavioural problems. In another definition, Ladd (1999) simply defined social skills as behaviours considered for improving positive relational consequences like acceptance by peers and friendship. And finally, social skill is a part of the broader concept of social capability (Gresham, 1981\&1982). which indicates mutual compliance with social environment and in relationship with age peers (Khanzadeh et al, 2013).

Gresham and Eliot (1990) defined social skills as acceptable behaviours learnted by the individuals, which pave the way for their interactions with others in the society. According to Yüksel, different dimensions developed for social behaviours have been based on the aforementioned definitions. The model evolved by Riggio (1986) is among the most acceptable models in the world. Riggio holds that several minor factors form the social behaviours. In the questionnaire of social skills designed based on Riggio's theory, six key dimensions have been identified, including: Emotional Expressivity, Emotional Sensitivity, Emotional Control, Social Expressivity, Social Sensitivity, Social Control (Aktı and Gurol, 2012).

Results of different analyses suggest that social skills are accompanied with considerable consequences in work environment as well as private life, including participation in social activities which can in turn lead to sentiments such as dependence, acceptance, and self-confidence. Social skills help the individual make relationships with others more easily, have more positive feelings about oneself, and better manage and lead a large project (2006, Pettry).

Johnson and Johnson (1999) mentioned six significant consequences of social skills. The first desirable consequence is personal and identity development of individuals because a major portion of people's identities are formed by their relationships with others. Additionally, social skills improve their productivity and chance of success on the way of their occupational progress. Social skills help the individuals collaborate easily, lead their subordinates, encounter intricate situations, and resolve work-related problems and issues.

Life quality is another positive consequence of social skills because every person needs friendly, good, and tight relations in his/her life. On the other hand, physical health is also promoted via positive and supportive relationships. It has been proved that establishment of positive relationships with others reduce psychological and mental burdens. Personal identity of individuals improves their ego, self-sufficiency, and independence. The last significant consequence 
of social skills is the ability to encounter stress. Supportive and positive relationships mitigate severity and level of stressful events and decrease the anxiety. Such relationships help people manage stressful situations with the aid of their information, resources, and feedbacks (Johnson and Johnson, 1999).

But, the concept of cultural capital has played an outstanding role in formation of different research works during the last four decades in various scientific fields such as sociology, history, cultural studies, and feministic projects. The attention focus of cultural capital is social inequality, as it emerged particularly in Bourdieu's well-known and international research $(1970,1984)$. During the recent years, great deal of attention has been paid to cultural capital (Devine, 2004; Skeggs, 1997; Savage, Barlu, Dickens and Fielding, 1992). As the great American sociologist Treiman (2002) has recently stated: cultural capital has been the most important cause of changes in social position within all western societies whereas these positions have been fixed and unchangeable in the past. Despite long history of such discussions about concept of cultural capital in research works on social and educational class, there remains still a profound gap in investigation of significance of cultural capital for comprehending cultural citizenship concept. This gap has deepened during the recent years with increased attention to development of cultural policies (Bennet \& Savage, 2004).

Bourdieu considers cultural capital as the dominant form of symbolic capital in the societies where the school system and writing art have well developed. In such societies, symbolic capital is characterized by stable features illustrated in the form of books, institutionalized titles such as educational certificates, and so on.

Cultural Capital: the ability to recognize and use cultural commodities in each person is referred to as "cultural capital" which encompasses consistent tendencies of the persons that are accumulated in them during socialization (Bourdieu, 2014:169). Cultural capital is defined as an asset that illustrates, stores, or provides cultural value in addition to any sort of economic value that it may have (Tarasebi, 2003: 92).

In Bourdieu's attitude, cultural capital can exist in three formsin social context: Embodied Capital, Objectified Capital, and Institutionalized Capital (Bourdieu, 1986).

According to the arguments above, the essential problem of the present research is to determine the effects of media literacy and its dimensions including critical thinking and media knowledge, analysis and reaction, and observed hidden messages on social skills via moderating effect of cultural capital. Taking into account the research problem, the principal hypotheses of the research are as below:

Hypothesis 1: The dimension of media knowledge (critical thinking) affects social skills, and, cultural capital moderates this effect.

Hypothesis 2: The dimension of analysis and reaction affects social skills, and, cultural capital moderates this effect.

Hypothesis 3: The dimension of observed hidden messages affects social skills, and, cultural capital moderates this effect.

Based on the principal hypotheses, the following conceptual model can be assumed for the major research hypothesis and objective:

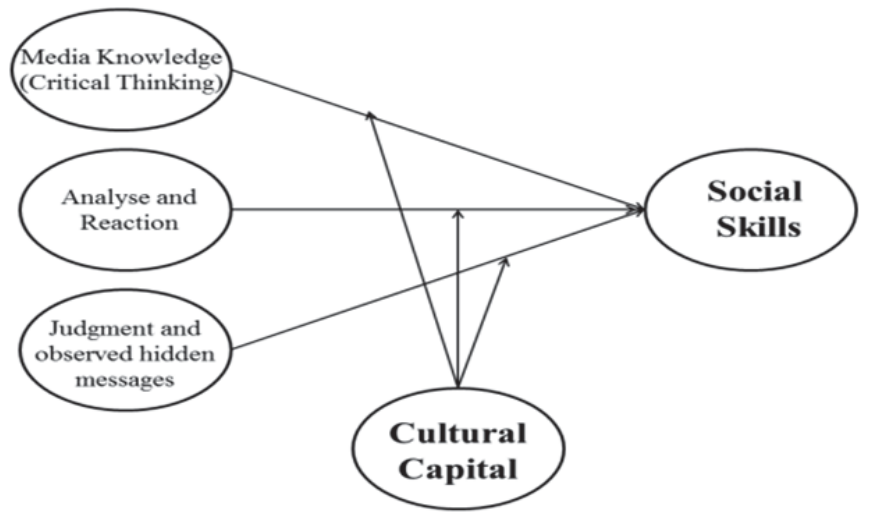

Figure (1): Conceptual model of research 


\section{Research Methodology}

To analyze research statements, the current section deals with research methodology as well as the evaluation tool and statistical population and sample. Since the present research is intended to propose a model for analyzing effects of media literacy components on social skills and also moderating effect of cultural capital based on structural equation modeling, it is regarded as an applied research in terms of objective and a correlation-type study in terms of data collection technique. The main data collection tool is questionnaire which comprised 98 questions based on the variables under analysis. The questions are composed of two parts: the first part includes questions for acquiring demographic information (consisting of 6 questions about gender, education level, marital status, age, the media used, and occupation), and the second part includes the technical questions in Likert scale used from the standard questionnaires as shown in Table (1).

Table (1): The questionnaire and the research variables under analysis

\begin{tabular}{|l|l|c|l|}
\hline $\begin{array}{l}\text { Variables under } \\
\text { analysis }\end{array}$ & $\begin{array}{l}\text { Dimensions and number of questions in the } \\
\text { questionnaire }\end{array}$ & $\begin{array}{c}\text { Total number of } \\
\text { questions }\end{array}$ & Remarks \\
\hline Social Skills & $\begin{array}{l}\text { Emotional Expressivity: Questions No. 18-21 } \\
\text { Emotional Sensitivity: Questions No. 22-25 } \\
\text { Emotional Control: Questions No. 26-28 } \\
\text { Social Sensitivity: Questions No. 29-32 } \\
\text { Social Control: Questions No. 33-36 } \\
\text { Social Expressivity: Questions No. 37-40 }\end{array}$ & 23 & $\begin{array}{l}\text { Standard Questionnaire of Social } \\
\text { Skills by Oldmeadow et al. (2013) }\end{array}$ \\
\hline Media Literacy & $\begin{array}{l}\text { Media knowledge (critical thinking): Questions No. 1-6 } \\
\text { Analysis and Reaction: Questions No. 8-12 } \\
\text { Observed Hidden Messages: Question No. 7 and 13-17 }\end{array}$ & \multirow{2}{*}{14} & $\begin{array}{l}\text { Standard Questionnaire of Media } \\
\text { Literacy by Karaman \& Karatash } \\
\text { (2009) }\end{array}$ \\
\hline Cultural Capital & $\begin{array}{l}\text { Institutionalized Capital: Questions No. 95-99 } \\
\text { Objectified Capital: Questions No. 41-66 } \\
\text { Embodied Capital: Questions No. 68-93 }\end{array}$ & $\begin{array}{l}\text { The questionnaire designed based } \\
\text { on Bourdieu's approach }\end{array}$ \\
\hline
\end{tabular}

To evaluate the reliability of the questionnaire, an initial sample of 30 questionnaires was tested, and then using the acquire data, reliability coefficient was calculated based on Cronbach's alpha method and was obtained equal to 0.876 , 0.896 , and 0.913 respectively for the questions related to media literacy, social skills, and cultural capital. These values indicate that the questionnaire is reliable enough. Factor analysis was deployed to assess validity of questions. In the present research, the questionnaires were checked using techniques of exploratory and confirmatory factor analyses. The results demonstrate that adequacy coefficient of the sample exceeds 0.5 and Bartlett's test results have significance level over 1.96. The results of this stage are included in Table (2). It shall be mentioned that some research questions were eliminated due to low commonality ratio and disproportionality in the factor structure.

Table (2): Results of exploratory and confirmatory factor analyses

\begin{tabular}{|c|c|c|c|}
\hline $\begin{array}{l}\text { Main Dimension and Total } \\
\text { Number of Quetions }\end{array}$ & $\begin{array}{l}\text { Name of factors in order of significance } \\
\text { explained by the variance }\end{array}$ & $\begin{array}{l}\text { The explained } \\
\text { variance }\end{array}$ & Confirmatory factor analysis results \\
\hline Media Literacy (14 questions) & $\begin{array}{l}\text { Media knowledge (critical thinking): } 21.117 \\
\text { Analysis and Reaction: } 20.446 \\
\text { Observed Hidden Messages: } 14.126\end{array}$ & 55.689 & $\begin{array}{l}\chi^{2} / \mathrm{df}=1.76, \mathrm{GFI}=0.96, \mathrm{AGFI}= \\
0.94, \mathrm{RMSEA}=0.037\end{array}$ \\
\hline Social Skills (23 questions) & $\begin{array}{l}\text { Emotional Expressivity: } 8.920 \\
\text { Emotional Sensitivity: } 8.642 \\
\text { Emotional Control: } 8.565 \\
\text { Social Sensitivity: } 8.234 \\
\text { Social Control: } 8.197 \\
\text { Social Expressivity: } 7.026\end{array}$ & 49.585 & $\begin{array}{l}\chi^{2} / \mathrm{df}=1.15, \mathrm{GFI}=0.96, \mathrm{AGFI}= \\
0.95, \mathrm{RMSEA}=0.016\end{array}$ \\
\hline Cultural Capital (55 questions) & $\begin{array}{l}\text { Institutionalized Capital: } 14.992 \\
\text { Objectified Capital: } 14.891 \\
\text { Embodied Capital: } 4.038\end{array}$ & 33.921 & $\begin{array}{l}\chi^{2} / \mathrm{df}=0.99, \mathrm{GFI}=0.98, \mathrm{AGFI}= \\
0.98, \mathrm{RMSEA}=0.001\end{array}$ \\
\hline
\end{tabular}




\subsection{Statistical Population and Sample of Research}

The statistical population under analysis in the present research is all citizens of Isfahan City in 2013.The city's population was declared to be 1,796,967 persons. To gain sufficient sample size for the present research, the researchers benefitted from Cochran's finite population sampling formula and the sample size was estimated as follows:

$$
n=\frac{N\left(t_{\alpha / 2}\right)^{2} s^{2}}{(N-1) \varepsilon^{2}+\left(t_{\alpha / 2}\right)^{2} s^{2}}=\frac{1796967(1.96)^{2}(0.5 * 0.5)}{(1796967-1)(.05)^{2}+(1.96)^{2}(0.5 * 0.5)}=384
$$

Therefore, samples were taken in the present research with respect to the estimated sample size based on Cochran's formula and proportional to each stratum, as presented in Table (3).

Table 3: Statistical sample size proportional to different regions of Isfahan City

\begin{tabular}{ccc}
\hline \hline Region & Region's Population & Sample Size of Region \\
\hline 1 & 79,966 & 25 \\
\hline 2 & 66,590 & 21 \\
\hline 3 & 111,889 & 35 \\
\hline 4 & 128,030 & 40 \\
\hline 5 & 161,543 & 51 \\
\hline 6 & 116,464 & 37 \\
\hline 7 & 151,191 & 48 \\
\hline 8 & 240,664 & 76 \\
\hline 9 & 74,336 & 24 \\
\hline 10 & 213,547 & 68 \\
\hline 11 & 59,555 & 19 \\
\hline 12 & 126,884 & 40 \\
\hline 13 & 121,032 & 38 \\
\hline 14 & 145,276 & 46 \\
\hline Sum & $1,796,967$ & 568 \\
\hline \hline
\end{tabular}

384 questionnaires were collected finally. The researcher personally went to different regions of Isfahan City for 3 months in order to distribute the questionnaires and gather the needed data. According to the collected data, the demographic information can be observed in Table (4).

Table (4): Demographic variables used in the research

\begin{tabular}{|c|c|c|c|}
\hline Variable & Frequency percentage & Variable & Frequency percentage \\
\hline \multicolumn{2}{|l|}{ Age } & \multicolumn{2}{|c|}{ Media used simultaneously } \\
\hline Below 24 years & 34.9 & Newspaper & 15.5 \\
\hline $25-30$ years & 43.5 & Book & 17.5 \\
\hline $31-35$ years & 16.9 & Magazine & 5.3 \\
\hline Over 35 years & 3.6 & Television & 41.6 \\
\hline No answer & 1.1 & Radio & 2.5 \\
\hline \multicolumn{2}{|l|}{ Education Level } & Internet & 26.9 \\
\hline Less than high-school diploma & 27.3 & No answer & 0.8 \\
\hline High-school diploma and associate degree & 23.2 & \multicolumn{2}{|c|}{ Occupation } \\
\hline Bachelors' degree & 36.7 & Employee & 49.2 \\
\hline Masters' degree & 12 & Unofficial Job & 6.8 \\
\hline $\mathrm{PhD}$ & 0.8 & Student & 34.1 \\
\hline \multicolumn{2}{|l|}{ Marital Status } & Others & 9.6 \\
\hline Single & 68.7 & No answer & 0.3 \\
\hline Married & 30.5 & \multicolumn{2}{|r|}{ Gender } \\
\hline \multirow[t]{2}{*}{ No answer } & 0.8 & Male & 67.6 \\
\hline & & Female & 32.4 \\
\hline
\end{tabular}




\subsection{Research Findings}

The research hypotheses were analyzed in explanation of inferential findings of the research. Structural equation modeling with moderation effect was applied since the research objective was to propose a model for analyzing effects of media literacy components on improvement of social skills and moderating impact of cultural capital.

Hypothesis 1: The dimension of media knowledge (critical thinking) affects social skills, and, cultural capital moderates this effect. Warp PLS software was used to verify the respective hypothesis; the software output is seen in Figure (2).

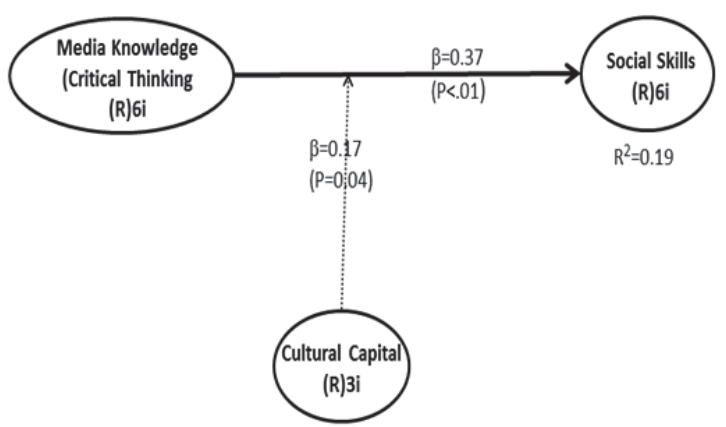

Figure (2) Model of relationships between variables in the first hypothesis

As shown in Figure (15-4), possession of media knowledge has an effect size of 0.37 at p-value of less than 0.05 on social skills and the effect is significant. On the other hand, size of moderating effect of cultural capital is 0.17 at $p$-value below 0.05 and the moderating effect is significant on the relationship between media knowledge and social skills. Table (5) summarizes the effects as follows.

Table (5): Indirect and total effects of latent variables of the first hypothesis

\begin{tabular}{lcc}
\hline \hline \multicolumn{2}{c}{ Indirect and total effects } \\
\hline \multicolumn{2}{c}{ Total effects } \\
\hline Social skills & 0.365 & 0.166 \\
\hline \hline
\end{tabular}

Now, a fundamental question arises: are the fit indexes of the model significant? For this purpose, the fit indexes of the model were analyzed as summarized in Table (6).

Table (6): Summary of coefficients of latent variables in the first hypothesis

\begin{tabular}{|c|c|c|c|c|c|}
\hline & \multicolumn{5}{|c|}{ Standard Value Cultural Capital Social Skills Media Knowledge Media Knowledge* Cultural Capital } \\
\hline R square coefficient & & & 0.187 & & \\
\hline Adjusted R square coefficient & & & 0.158 & & \\
\hline Composite reliability & $0.7 \leq$ & 0.712 & 0.857 & 0.897 & 0.861 \\
\hline Cronbach's alpha & $0.7 \leq$ & - & 0.798 & 0.859 & 0.842 \\
\hline Average variance extracted & $0.5 \leq$ & 0.51 & 0.507 & 0.596 & 0.583 \\
\hline VIF & $5 \geq$ & 1.281 & 1.382 & 1.195 & 1.161 \\
\hline $\mathrm{Q}^{2}$ index & $0.15 \leq$ & & 0.194 & & \\
\hline Goodness of fit index & $0.25 \leq$ & & 0.293 & & \\
\hline
\end{tabular}

All above mentioned indexes are significant and corroborate suitable fit of model. Furthermore, divergent validity of the respective model was also investigated as illustrated in Table (7). 
Table (7): Divergent validity related to the first hypothesis model

\begin{tabular}{lcccc}
\hline \hline & Media Knowledge & Social Skills & Cultural Capital & Media Knowledge* Cultural Capital \\
\hline Media Knowledge & $(0.112)$ & & & \\
\hline Social Skills & 0.299 & $(0.713)$ & & \\
\hline Cultural Capital & 0.089 & 0.436 & $(0.657)$ & $(0.547)$ \\
\hline Media Knowledge* Cultural Capital & -0.319 & -0.172 & 0.094 & \\
\hline \hline
\end{tabular}

As the square root of the extracted variance (inside the parenthesis and on the leading diagonal of matrix) is larger than the factor loadings of all latent variables in each column, the model of the first research hypothesis enjoys suitable divergent validity.

According to what mentioned earlier, the first hypothesis of the research asserting "the dimension of media knowledge (critical thinking) affects improvement of social skills among Isfahanian citizens, and, cultural capital moderates this effect" is confirmed. This result is illustrated in Figure (3).
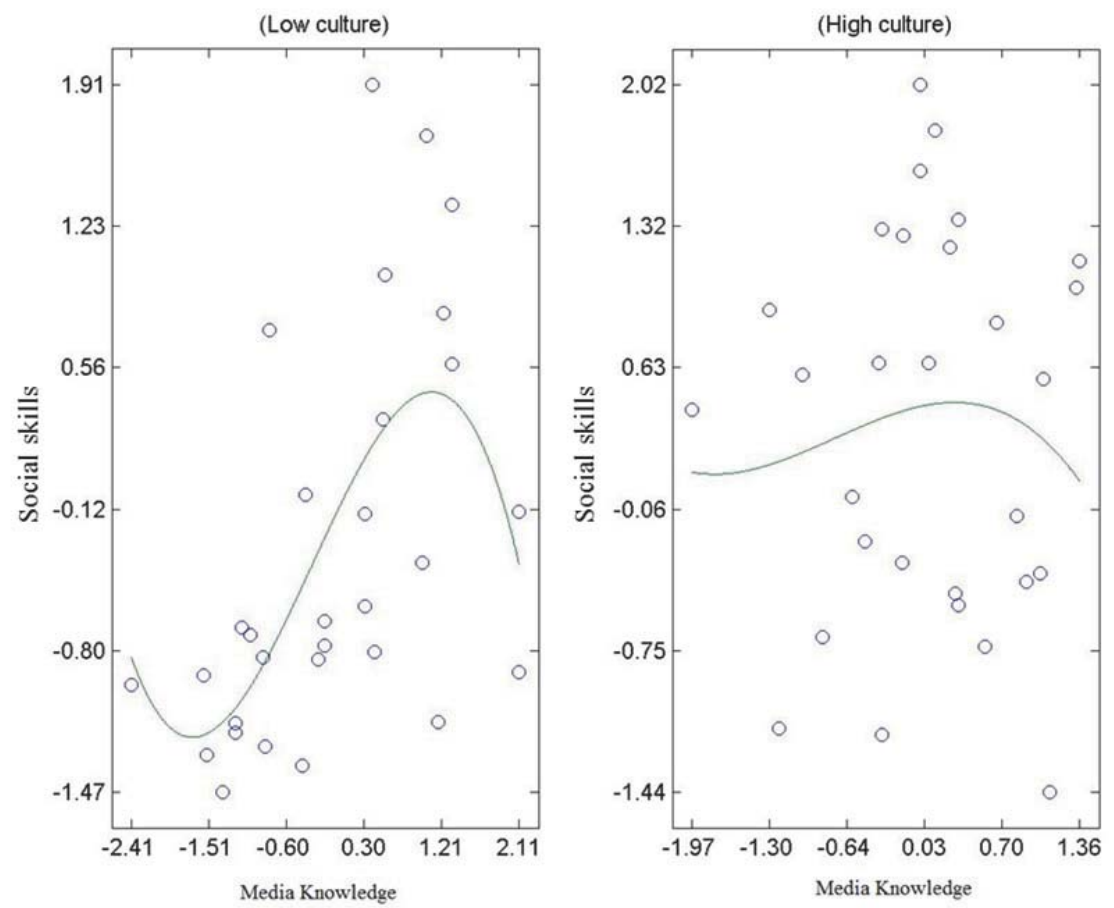

Figure (3): Separate moderation diagrams with the coordinates of information related to variables of the first hypothesis

As illustrated in Figure (3), social skills increase with an increase in level of media knowledge and this relationship is moderated by cultural capital. It means when cultural capital is high, compared to the situation where cultural capital is low, increase of media knowledge level leads to further enhancement of social skills.

Hypothesis 2: The dimension of analysis and reaction affects social skills, and, cultural capital moderates this effect. Warp PLS software was used to verify the respective hypothesis; the software output is seen in Figure (5). 


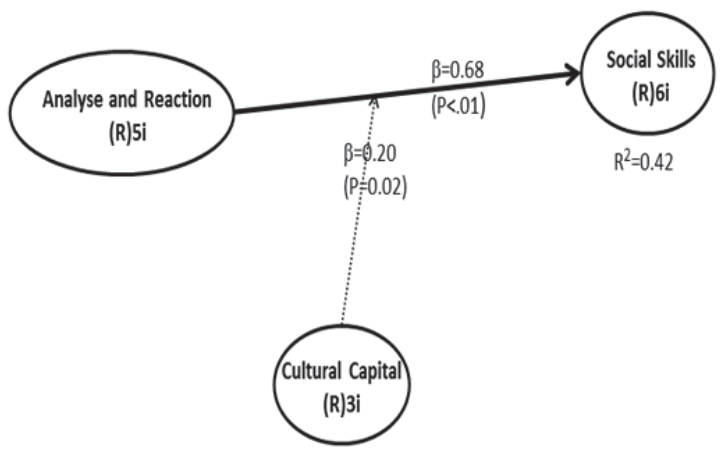

Figure (4): Model of relationships between variables of the second hypothesis

As illustrated in Figure (4), analysis and reaction has an effect size of 0.68 on social skills at p-value $\leq 0.05$ and this effect is significant. Furthermore, size of moderating effect of cultural capital is 0.2 at p-value of 0.02 which is less than 0.05 , indicating that cultural capital significantly affects analysis and reaction as well as social skills. Table (8) includes the respective effects in summary.

Table (8): Indirect and total effects of variables in second hypothesis

\begin{tabular}{ccc}
\hline \hline \multicolumn{2}{c}{ Indirect and total effects } \\
\hline \multicolumn{2}{c}{ Total effects } \\
\hline & Analysis and Reaction & Analysis and Reaction ${ }^{\star}$ Cultural Capital \\
\hline Social skills & 0.676 & 0.199 \\
\hline \hline
\end{tabular}

An essential question is posed at this step: are fit indexes of model significant? For this purpose, the suitability indexes for fit of model are analyzed as summarized in Figure (9).

Table (9): Values of coefficients of latent variables in the second hypothesis

\begin{tabular}{lccccc}
\hline \hline & $\begin{array}{c}\text { Standard } \\
\text { Value }\end{array}$ & $\begin{array}{c}\text { Analysis and } \\
\text { Reaction }\end{array}$ & $\begin{array}{c}\text { Social } \\
\text { Skills }\end{array}$ & $\begin{array}{c}\text { Cultural } \\
\text { Capital }\end{array}$ & $\begin{array}{c}\text { Analysis and Reaction* } \\
\text { Cultural Capital }\end{array}$ \\
\hline R square coefficient & & & & 0.417 & \\
\hline Adjusted R square coefficient & & & 0.397 & 0.767 \\
\hline Composite reliability & $0.7 \leq$ & 0.772 & 0.706 & 0.857 & 0.731 \\
\hline Cronbach's alpha & $0.7 \leq$ & 0.710 & 0.712 & 0.798 & 0.209 \\
\hline Average variance extracted & $0.5 \leq$ & 0.512 & 0.547 & 0.508 & 1.038 \\
\hline VIF & $5 \geq$ & 1.784 & 1.305 & 1.823 & 0.496 \\
\hline$Q^{2}$ index & $0.15 \leq$ & & 0.404 & \\
\hline Goodness of fit index & $0.25 \leq$ & & & & \\
\hline \hline
\end{tabular}

As illustrated in Figure (9), all aforementioned indexes are significant and reflect suitable model fit. In addition, divergent validity of the model shall be also analyzed as shown in Table (10).

Table (10): Divergent validity related to the model of second hypothesis

\begin{tabular}{|c|c|c|c|}
\hline & Analysis and Reaction Cultural Capita & Social Skills & eaction * Cultural Capital \\
\hline Analysis and Reaction & $(0.645)$ & & \\
\hline Social Skills & $(0.657)$ & & \\
\hline Cultural Capital & 0.436 & $(0.713)$ & \\
\hline Analysis and Reaction * Cultural Capital & 0.166 & 0.057 & $(0.475)$ \\
\hline
\end{tabular}


Taking into account the fact that square root of the extracted variance (inside the parenthesis and on the leading diagonal of matrix) is larger than the factor loadings of each latent variable in each column of Table (10), the model of second research hypothesis has the adequate divergent validity.

Considering the former discussions, the second research hypothesis stating "dimension of analysis and reaction of media literacy affects social skills of Isfahanian citizens and cultural capital moderates this effect" is confirmed, as illustrated in Figure (5).
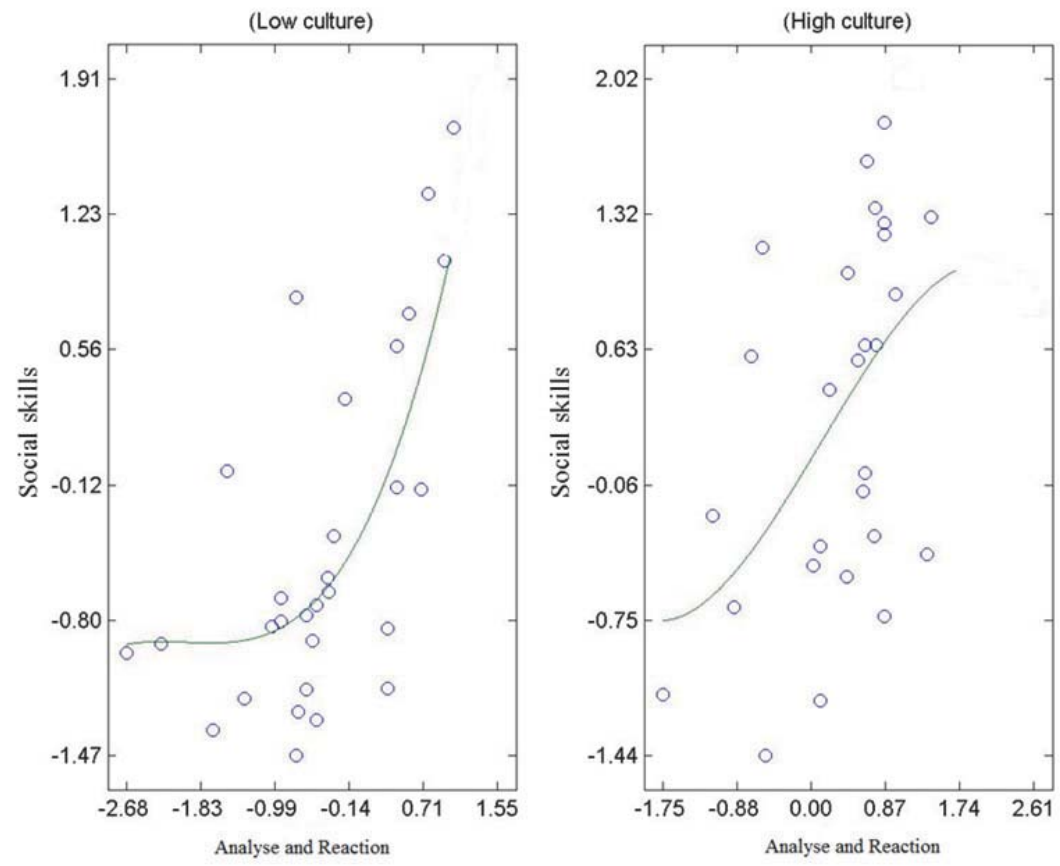

Figure (5): Separate moderation effects with coordinates of information related to second hypothesis variables

As manifested in the figure, social skills are improved with further increase in analysis and reaction and this relationship is moderated by cultural capital i.e. when cultural capital is high, increase in the level of analysis and reaction leads to further improvement of social skills.

Hypothesis 3: The dimension of observed hidden messages affects social skills, and, cultural capital moderates this effect. Warp PLS software was used to analyze the abovementioned hypothesis, and the output can be observed in Figure (6).

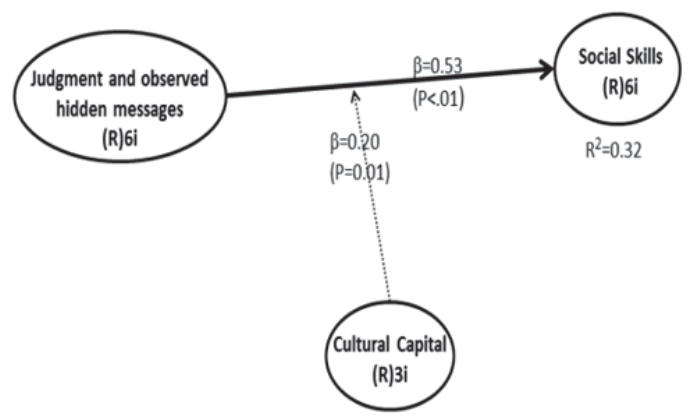

Figure (6): Model of relationships between variables of the third hypothesis 
As demonstrated in Figure (6), the dimension of observed hidden messages has an effect size of 0.53 on social skills at p-value of 0.01 which is smaller than 0.05 and this effect is significant. On the other hand, size of moderating effect of cultural capital is 0.2 at p-value of 0.01 which is less than 0.05 and has a significant moderating effect on observed hidden messages and social skills. Table (11) summarizes the respective effects.

Table (11) Indirect and total effects of all non-latent variables of the third hypothesis

\begin{tabular}{ccc}
\hline \hline \multicolumn{2}{c}{ Indirect and total effects } \\
\hline \multicolumn{2}{c}{ Total effects } \\
\hline & Observed Hidden Messages & Observed Hidden Messages ${ }^{\star}$ Cultural Capital \\
\hline Social Skills & 0.526 & 0.204 \\
\hline \hline
\end{tabular}

Now the essential question is: are the model fit indexes significant? For this purpose, suitability indexes of model fit are analyzed as summarized in Figure (12).

Table (12) Summary of coefficients of latent variables of the third hypothesis

\begin{tabular}{lccccc}
\hline \hline & $\begin{array}{c}\text { Standard } \\
\text { Value }\end{array}$ & $\begin{array}{c}\text { Observed Hidden } \\
\text { Messages }\end{array}$ & $\begin{array}{c}\text { Social } \\
\text { Skills }\end{array}$ & $\begin{array}{c}\text { Cultural } \\
\text { Capital }\end{array}$ & $\begin{array}{c}\text { Observed Hidden } \\
\text { Messages* Cultural Capital }\end{array}$ \\
\hline R square coefficient & \multicolumn{1}{c}{0.317} & & \\
\hline Adjusted R square coefficient & & 0.293 & & 0.795 \\
\hline Composite reliability & $0.7 \leq$ & 0.881 & 0.875 & 0.765 & 0.807 \\
\hline Cronbach's alpha & $0.7 \leq$ & 0.834 & 0.797 & 0.745 & 0.503 \\
\hline Average variance extracted & $0.5 \leq$ & 0.559 & 0.508 & 0.561 & \\
\hline VIF & $5 \geq$ & 1.221 & 1.43 & 1.316 & \\
\hline$Q^{2}$ index & $0.15 \leq$ & & 0.341 & & \\
\hline Goodness of fit index & $0.25 \leq$ & 0.347 & & \\
\hline \hline
\end{tabular}

All abovementioned indexes are significant and indicate suitable model fit. Additionally, divergent validity of the model shall be also examined as shown in Table (13).

Table (13): Divergent validity related to the third hypothesis model

\begin{tabular}{lcccc}
\hline \hline & $\begin{array}{c}\text { Observed Hidden } \\
\text { Messages }\end{array}$ & $\begin{array}{c}\text { Social } \\
\text { Skills }\end{array}$ & $\begin{array}{c}\text { Cultural } \\
\text { Capital }\end{array}$ & $\begin{array}{c}\text { Observed Hidden } \\
\text { Messages }{ }^{*} \text { Cultural Capital }\end{array}$ \\
\hline Observed Hidden Messages & $(0.748)$ & & & \\
\hline Social Skills & 0.41 & $(0.713)$ & & \\
\hline Cultural Capital & 0.213 & 0.436 & $(0.657)$ & \\
\hline Observed Hidden Messages ${ }^{*}$ Cultural Capital & -0.026 & 0.163 & 0283 & $(0.519)$ \\
\hline \hline
\end{tabular}

According to Table (13), it can be seen that the square root of the extracted variance (inside the parenthesis and on the principal diagonal of the matrix) is larger than factor loadings of each of latent variables in each column. The third hypothesis model has the suitable divergent validity.

Based on the earlier discussions, the third research hypothesis stating "dimension of observed hidden messages of medial literacy affects social skills of Isfahanian citizens, and, cultural capital moderates this effect" is confirmed, as illustrated in Figure (7). 

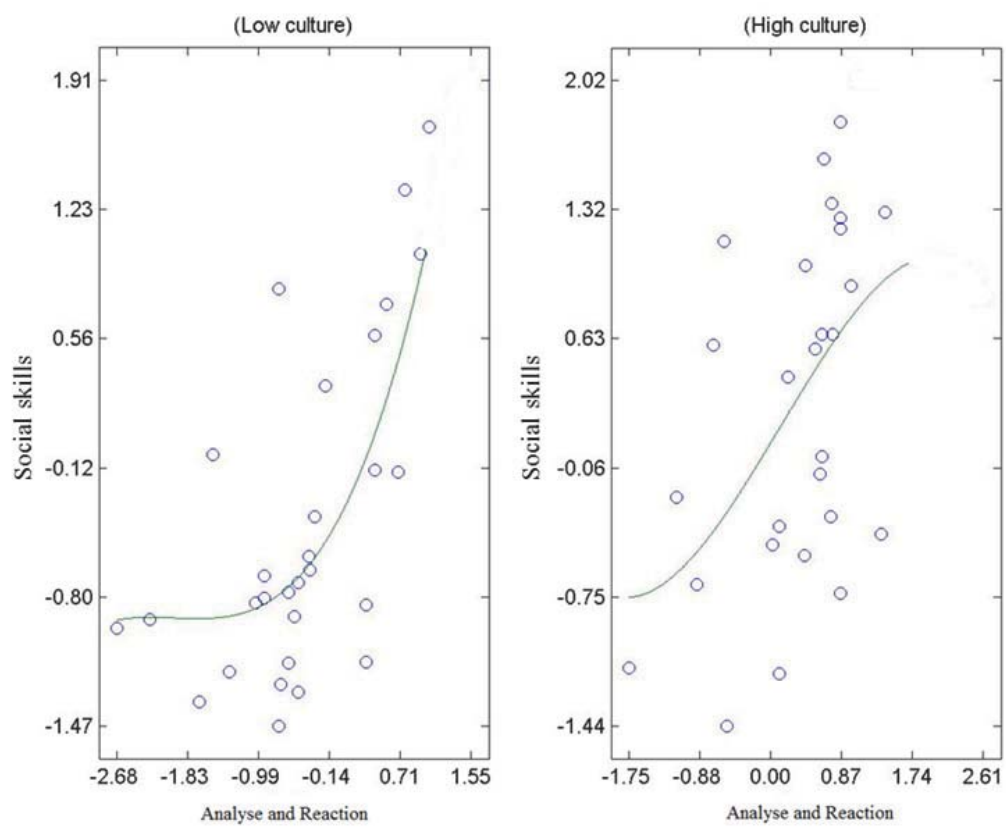

Figure (7) Separate moderation diagrams with coordinates of information related to the variables of the third hypothesis

As shown in Figure (7), social skills improve with increase in the dimension of observed hidden messages and this relationship is moderated by cultural capital i.e. increase in dimension of observed hidden messages results in further improvement of social skills when cultural capital is high.

\section{Conclusions}

In the present paper, the data were first analyzed using descriptive statistics such as frequency tables and diagrams, and then, research hypotheses were verified based on inferential statistics and using Warp PLS software. The acquired results are summarized as below:

\subsection{First Hypothesis:}

According to the results presented in Tables (5) through (7) as well as Figures (2) through (4), the first research hypothesis asserting "media knowledge dimension of media literacy affects social skills, and, cultural capital moderates this effect" is significant with an effect size of 0.37 and cultural capital moderates the relationship between media literacy and social skills with an effect size of 0.17 .

The results of the present research are not in agreement with any other research work in which effects of all three variables have been analyzed. However, in terms of effect of media knowledge (critical thinking) dimension of media literacy on improvement of social skills, the research results are in agreement with those acquired in the research carried out by Zelsdorf (2014) and Oktai \& Guruol (2012). They also reached to the conclusion that media literacy, including the critical thinking dimension, is significantly correlated to improvement of social skills of individuals but moderating effect of cultural capital was not considered in their research.

In interpretation of the respective hypothesis, it can be stated that the individual gradually gains the capability of logical thinking and becomes able to critique the messages when media knowledge increases. This results in improvement of social skills with increase of individual's media knowledge. But, when cultural capital is involved in the relationship between these two variables, the results are indicative of moderating effect of cultural capital. It means when the cultural capital of individuals is high, as compared to the state when the cultural capital of individuals is low, increase 
in the level of media knowledge leads to further improvement of social skills. It is because of the fact that cultural capital consists of three dimensions of objectified, embodied, and institutionalized capitals. As a result, the people with higher cultural capital achieve more coherent and powerful critical thinking by the course of time in comparison with those having lower cultural capital. This results in reinforcement of social sensitivity of individuals to social events as well as their social control and social expressivity, in turn leading to strengthening of social skills.

\subsection{Second Hypothesis}

The analysis and reaction dimension of media literacy affects social skills of Isfahanian citizens, and, cultural capital moderates this effect. According to the results presented in Tables (8) through (10) and also Figures (5) through (7), the second research hypothesis asserting effect of analysis and reaction dimension of media literacy on social skills and also moderating effect of cultural capital on this relationship is confirmed. The effect size of the first relationship is equal to 0.68 and size of moderating effect of cultural capital on this significant relationship is 0.2 .

The results of the present research are not in agreement with any other research work in which effects of all three variables have been analyzed. The research results, however, are in alignment with those obtained by Aktai \& Gouroul (2010). They concluded that analysis and reaction dimension affects on social skills but didn't take into account moderating effect of cultural capital.

In interpretation of the respective hypothesis, it can be stated that when the individuals gain the ability to analyze the media messages and media literacy is strengthened by this dimension, their social sensitivity, social control, and social expressivity are improved and strengthened in social skills while cultural capital can play the moderator role in the relationship between these two variables. It means when the individuals benefit from higher level of cultural capital, their embodied, objectified, and institutionalized capitals lead to more rapid reinforcement of their ability to analyze media messages, resulting in larger impact on social skills in comparison with the cases where the individuals have lower level of cultural capital.

\subsection{Third Hypothesis}

Observed hidden messages dimension of media literacy affects on social skills and cultural capital moderates this effect. According to the results presented in Tables (11) through (13) and also Figures (8) through (13), the third research hypothesis asserting "observed hidden messages dimension of media literacy affects on social skills and cultural capital moderates this relationship" is confirmed. The effect size of the main relationship is equal to 0.53 and the size of moderating effect of cultural capital on this significant relationship is 0.2 .

The results of the present research are not in agreement with any other research work in which effects of all three variables have been analyzed. The research results, however, are in alignment with those obtained by Aktai \& Gouroul (2012). regarding the relationship between observed hidden messages dimension of media literacy and social skills. Nonetheless, they didn't take into account moderating effect of cultural capital.

Based on the research results, it can be interpreted that when the ability to judge, critique and analyze hidden messages of media is enhanced in the individuals, their social control, emotional sensitivity, and emotional control are influenced and social skills are improved. But, as the individuals having higher embodied, objectified, and institutionalized capitals can gain the ability to judge and analyze the hidden media messages at higher speed, therefore their social skills are improved more rapidly as well. On contrary, low level of cultural capital causes the individuals to more slowly acquire the ability to analyze, critique, and judge the media messages resulting in deceleration of acquisition of social skills in the individual.

Keeping in mind the research results, the research recommendations are proposed as follows:

\section{Research Recommendations}

Before proposing the practical recommendations, it must be mentioned that the current study had the following restrictions that shall be taken into account by the future researches. The major restrictions included:

The present research was carried out among residents of Isfahan City, and since different results might be achieved by conducting the research in another statistical population, utmost care must be devoted in generalizing the results to other populations.

In addition, there are many variables out of researchers' controls that might influence the relationship between media literacy and social skills. The examples are cultural circumstances of population, effects of individuals' characters 
on the responses to the questions, and the discrepancies associated with demographic variables. Hence, the respective variables and particularly the cultural variables might restrict the research results.

Based on the research results, the following solutions can be therefore recommended to improve the status of media literacy, social skills, and cultural capital:

- As a pervasive medium, television is recommended to prepare programs on instruction of media literacy and promotion of people's social skills and to broadcast them at appropriate time intervals.

- Analogous to the measures taken in well-developed countries like Canada, Japan, USA, Australia, and so on where media literacy has been incorporated as a training program in the course contents of school students, commensurate courses are also recommended to be prepared in educational system of Iran and taught for strengthening and improving media literacy of students.

- Macroscale and comprehensive planning shall be made for media-related actions in national and provincial networks and movement toward development of media knowledge in the whole country.

- Social skills shall be instructed to children at early ages of their life via families, kindergardens, and, children's skills of social control, social expressivity, and social sensitivity shall be promoted to improve their personal and relational skills.

- Cultural advertisements to be considered via mass communication media including television, radio, social networks, and so on for spreading the culture of studying and also enhancing the general knowledge of people because one of the most important reasons for underdevelopment of cultural and social-relational skills and failure in development of cultural capital is weakness in culture of studying. This issue was completely reflected in demographic studies of the research as well.

Instructional courses of critical thinking in society shall be taken into account for all age groups ranging from children to adults via media, schools, and scientific-cultural seminars.

Research works shall be carried out in order to identify media consumption regime of Isfahan City residents and to design an instructional program commensurate with the optimal media consumption regime.

Skills of social, political, and economic analyses of different media contents shall be considered by the relevant institutions and organizations.

Measures have to be taken for holding regular and perpetual tehncial forums and conferences related to media literacy by the institutes in charge of culture such as Ministry of Education, cultural centers of municipalities like culture houses, and local bureus of Ministry of Culture and Islamic Guidance.

\section{References}

Aktı S, Gurol A. 2012. Determining the Relationship between Media Literacy and Social Skills. Journal of Procedia - Social and Behavioral Sciences, 64: $238-243$.

Arjmand Gh. 2005. Media Literacy in Dialogue with Dr Gholam Reza Arjmand. Journal of Development in Training of Social Sciences, 4: 1-10.

Banai S. 2006. Our Lives In Turn of Media. Available from: http://www.hamshari.online.ir/news /?id=7909 . [Accessed 10 January 2015].

Bennett T, Savage M. 2004. Introduction: Cultural Capital and Cultural Policy. Journal of Cultural Trends, 50: 7-14.

Bourdieu P. 1986. The Forms of capital. Handbook of Theory and Research for the Sociology of Education, 1: 15-29.

Bourdieu P. 2014. Action Theory: Practical Reasons for Rational Selection. Translated by Mardiha M. 3th edn. Tehran: Nagsh O' Negar Publications, $344 \mathrm{p}$.

Francis G, McMullen V, Blue-Banning M, Haines S .2013. Increasing the social skills of a student with Autism through a literacy-Based Behavioral Intervention. Journal of Intervention in School and Clinic, 49:77-83.

Johnson D, Johnson R. 1999. Learning Together and Alone: Cooperative, Competitive, and Individualistic Learning. First edn, Boston: Allyn and Bacon, $526 \mathrm{p}$.

Karaman K, Karatash A. 2009.Media Literacy Levels of the Candidate Teachers. Journal of Elementary Education Online, 3: 798-808.

Khanzadeh A, Beh Pajoh A, Afrooz Gh, Mirzabeigi M. 2013. Identifying Social Skills Needed for Mentally Retarded Students for Course Planning of Social Skills. Journal of Psychological Studies, 1: 39-52.

Oldmeadow JA, Quinn S, Kowert R. 2013. Attachment style, social skills, and Facebook use amongst adults. Journal of Computers in Human Behavior, 29: 1142-1149.

Pettry W. 2006. Building Social Skills Through Activities, Certified Therapeutic Recreation Specialist. Available from http://www.DannyPettry.com. [Accessed 6 July 2006].

Terasbi D. 2003. Culture and Economic. Translated by Farhadi K. 1th edn, Tehran: Nei Publications. 256 p.

Zelsdorf E. 2014. Social Media, Social skills and well-Being: The impact of web 2.0. MSc thesis, University of Auckland. 